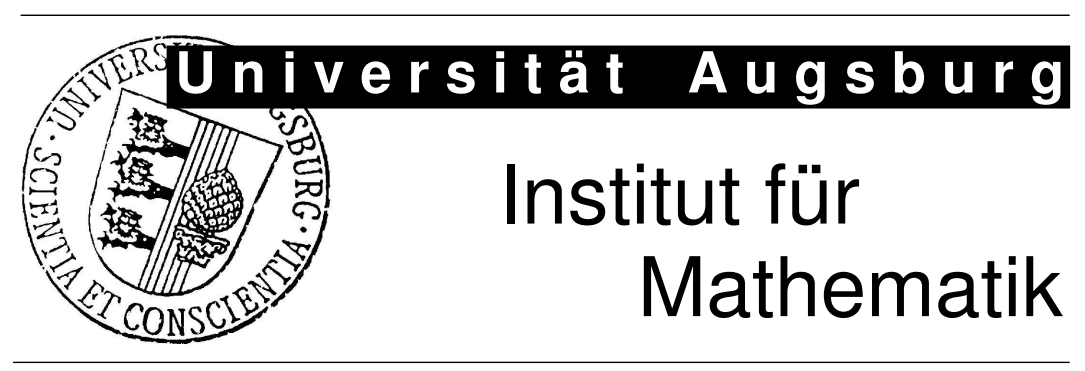

Christian Bräu, Lothar Heinrich

Mixing Properties of Stationary Poisson Cylinder Models 


\section{Impressum:}

\section{Herausgeber:}

Institut für Mathematik

Universität Augsburg

86135 Augsburg

http://www . math. uni-augsburg.de/de/forschung/preprints.html

\section{$V i S d P$ :}

Lothar Heinrich

Institut für Mathematik

Universität Augsburg

86135 Augsburg

Preprint: Sämtliche Rechte verbleiben den Autoren (C) 2016 


\title{
Mixing Properties of Stationary Poisson Cylinder Models
}

\author{
Christian Bräu and Lothar Heinrich ${ }^{1}$
}

\begin{abstract}
We study a particular class of stationary random closed sets in $\mathbb{R}^{d}$ called Poisson $k$ cylinder models (short: P-k-CM's) for $k=1, \ldots, d-1$. We show that all P-k-CM's are weakly mixing and possess long-range correlations. Further, we derive necessary and sufficient conditions in terms of the directional distribution of the cylinders under which the corresponding $\mathrm{P}-k-\mathrm{CM}$ is mixing. Regarding the $\mathrm{P}-(d-1)-\mathrm{CM}$ as union of "thick hyperplanes" which generates a stationary process of polytopes we prove that the distribution of the polytope containing the origin does not depend on the thickness of the hyperplanes.

Keywords : RANDOM CLOSED SET, HITTING FUNCTIONAL, RANDOM $k$-CYLINDER, INDEPENDENTLY MARKED POISSON PROCESS, TAIL $\sigma$-ALGEBRA, TYPICAL CELL, ZERO CELL

AMS 2010 MSC : Primary: 60D05 , 37A25; SECondary: 60G55 , 60G60
\end{abstract}

\section{Introduction and Preliminaries}

A stationary Poisson $k$-cylinder model (short: $\mathrm{P}-k-\mathrm{CM}$ ) in the $d$-dimensional Euclidean space $\mathbb{R}^{d}$ (for $d \geq 2$ and some $k \in\{1, \ldots, d-1\}$ ) is defined as union of randomly dilated $k$-flats whose individual spatial extensions, positions and directions are determined by a stationary independently marked Poisson process on $\mathbb{R}^{d-k}$. In this way a random closed set (short: RACS) in $\mathbb{R}^{d}$ with positive volume fraction (if the cylinder base in $\mathbb{R}^{d-k}$ has positive volume) is obtained which allows explicite formulas for a number of characteristics, e.g. $n$-point probabilities for any $n \in \mathbb{N}=\{1,2, \ldots\}$, see [20]. Although Poisson cylinder models have been considered already at the very beginning of the systematic study of RACSs, see [16] for $k=d-1$, [17] for any $k \in\{0,1, \ldots, d-1\}$, and [5] for stereological relationships, their importance as welltractable model in stochastic geometry with interesting properties (partly in contrast to the

\footnotetext{
${ }^{1}$ Institute of Mathematics, University of Augsburg, 86135 Augsburg, Germany

Corresponding author: E-mail: heinrich@math.uni-augsburg.de,
} 
frequently used Boolean model) was recognized just recently, see [20] and [8], [10] for central limit theorems of the volume and surface content in expanding windows.

To be precise, some further notation is needed. In stochastic geometry, a $k$-cylinder in $\mathbb{R}^{d}$ is defined as Minkowski sum $B \oplus \mathbb{L}$ of a direction space $\mathbb{L} \in \mathcal{G}(d, k)$ (= the Grassmannian of $k$-dimensional subspaces of $\mathbb{R}^{d}$ ) and a compact base $B$ in the orthogonal complement $\mathbb{L}^{\perp}$, see e.g. [19] or [20]. In the following we go along the line suggested in [8], [10] (which slightly differs from that in [14] and [20]) and identify $\mathbb{L}$ with a unique element $O_{\mathbb{L}}$ of the equivalence class $\mathbf{O}_{\mathbb{L}}$ of orthogonal matrices $O \in \mathbb{S O}_{d}$ (i.e. $O \in \mathbb{R}^{d \times d}, O^{T}=O^{-1}$ and $\operatorname{det}(O)=1$ ) satisfying $O \mathbb{E}_{k}=\mathbb{L}\left(\right.$ and $\left.O \mathbb{E}_{k}^{\perp}=\mathbb{L}^{\perp}\right)$, where $\mathbb{E}_{k}=\operatorname{span}\left\{e_{d-k+1}, \ldots, e_{d}\right\}, \mathbb{E}_{k}^{\perp}=\operatorname{span}\left\{e_{1}, \ldots, e_{d-k}\right\}$ for $k=1, \ldots, d-1$ with the usual orthonormal basis $\left\{e_{1}, \ldots, e_{d}\right\}$ of $\mathbb{R}^{d}$. In other words, two matrices $O_{1}, O_{2}$ belong to the compact set $\mathbf{O}_{\mathbb{L}} \subset \mathbb{S O}_{d}$ iff $O_{1}^{T} O_{2}$ belongs to the set of orthogonal block matrices $\mathbb{S}\left(\mathbb{O}_{d-k} \times \mathbb{O}_{k}\right)$ defined by

$$
\left\{\left(\begin{array}{cc}
A & 0 \\
0 & B
\end{array}\right): A \in \mathbb{R}^{(d-k) \times(d-k)}, B \in \mathbb{R}^{k \times k}, A^{T}=A^{-1}, B^{T}=B^{-1}, \operatorname{det}(A)=\operatorname{det}(B)\right\} .
$$

The element $O_{\mathbb{L}}$ can be chosen in a canonical way, e.g. as lexicographically smallest element of the set of matrices $\mathbf{O}_{\mathbb{L}}$. In this way we get a one-to-one correspondence between $\mathbb{S O}_{d, k}=$ $\left\{O_{\mathbb{L}}:=\operatorname{lex} \min \mathbf{O}_{\mathbb{L}}: \mathbb{L} \in \mathcal{G}(d, k)\right\}$ and $\mathcal{G}(d, k)$ up to orientation of the subspaces. Note that for $k=1$ (and analogously for $k=d-1$ ) the orthogonal matrix $O_{\mathbb{L}}$ can be chosen such that $\operatorname{det}\left(O_{\mathbb{L}}\right)=1$ and $O_{\mathbb{L}} e_{d}=u$, where $u \in S^{d-1}:=\left\{x \in \mathbb{R}^{d}:\|x\|=1\right\}$ is expressed in terms of spherical coordinates and $u$ and $-u$ are identified. For example, in the special cases $\mathbb{L}=$ $\operatorname{span}\left\{(\cos \vartheta, \sin \vartheta)^{T}\right\} \in \mathcal{G}(2,1)$ and $\mathbb{L}=\operatorname{span}\left\{\left(\cos \vartheta_{1} \sin \vartheta_{2}, \sin \vartheta_{1} \sin \vartheta_{2}, \cos \vartheta_{2}\right)^{T}\right\} \in \mathcal{G}(3,1)$ we take the matrices

$$
O_{\mathbb{L}}(\vartheta)=\left(\begin{array}{cc}
\sin \vartheta & \cos \vartheta \\
-\cos \vartheta & \sin \vartheta
\end{array}\right) \text { and } O_{\mathbb{L}}\left(\vartheta_{1}, \vartheta_{2}\right)=\left(\begin{array}{ccc}
\sin \vartheta_{1} & \cos \vartheta_{1} \cos \vartheta_{2} & \cos \vartheta_{1} \sin \vartheta_{2} \\
-\cos \vartheta_{1} & \sin \vartheta_{1} \cos \vartheta_{2} & \sin \vartheta_{1} \sin \vartheta_{2} \\
0 & -\sin \vartheta_{2} & \cos \vartheta_{2}
\end{array}\right) \text {, }
$$

respectively, for $0 \leq \vartheta<\pi$ and $\left(\vartheta_{1}, \vartheta_{2}\right) \in[0,2 \pi) \times[0, \pi / 2]$. In the particular case $\mathbb{L}=\operatorname{span}\left\{\left(\cos \vartheta_{1} \cos \vartheta_{2}, \sin \vartheta_{1} \cos \vartheta_{2},-\sin \vartheta_{2}\right)^{T},\left(-\sin \vartheta_{1}, \cos \vartheta_{1}, 0\right)^{T}\right\} \in \mathcal{G}(3,2)$ it is easily checked that $O_{\mathbb{L}}^{*}\left(\vartheta_{1}, \vartheta_{2}\right) \mathbb{E}_{2}=\mathbb{L}$, where $O_{\mathbb{L}}^{*}\left(\vartheta_{1}, \vartheta_{2}\right)$ is obtained from $O_{\mathbb{L}}\left(\vartheta_{1}, \vartheta_{2}\right)$ by multiplying the first column of $O_{\mathbb{L}}\left(\vartheta_{1}, \vartheta_{2}\right)$ by -1 and exchanging it with the third column.

In this way, to each random subspace $\mathbb{L} \in \mathcal{G}(d, k)$ corresponds a (unique) random matrix $\Theta=\Theta(\mathbb{L}) \in \mathbb{S O}_{d, k}$ and vice versa. Throughout this paper, all random elements are defined on a common probability space $[\Omega, \sigma(\Omega), \mathbf{P}]$ and $\mathbf{E}$ denotes the expectation with respect to $\mathbf{P}$. Let $Q_{d, k}$ be a distribution on the Borel- $\sigma$-algebra of the mark space $\mathbb{M}_{d, k}:=\mathbb{S O}_{d, k} \times \mathcal{K}_{d-k}^{\prime}$, where $\mathcal{K}_{d-k}^{\prime}$ is the space of all non-empty compact sets in $\mathbb{R}^{d-k}$ equipped with the Hausdorff 
metric, see e.g. [14]. For later use, we put $\mathcal{K}_{d}:=\mathcal{K}_{d}^{\prime} \cup\{\emptyset\}$ and denote by $\mathcal{C}_{d}$ the subfamily of convex sets in $\mathcal{K}_{d}$, whereas $\mathcal{B}_{d}$ signifies the Borel- $\sigma$-algebra generated by the family $\mathcal{F}_{d}$ of all closed in $\mathbb{R}^{d}$. Further, let $\mathbf{o}_{\ell}$ flag the origin (null vector) in $\mathbb{R}^{\ell}$ for $\ell \geq 1$.

Now, we are ready to introduce a stationary independently marked Poisson point process (see e.g. [3],[9], [19]) on $\mathbb{R}^{d-k}$ with mark space $\mathbb{M}_{d, k}$, intensity $\lambda>0$ and mark distribution $Q_{d, k}$ as locally bounded counting measure $\Pi_{\lambda, Q_{d, k}}=\sum_{i \geq 1} \delta_{\left[X_{i},\left(\Theta_{i}, \Xi_{i}\right)\right]}$ on the product space $\mathbb{R}^{d-k} \times \mathbb{M}_{d, k}$, i.e., for some random element $\left(\Theta_{0}, \Xi_{0}\right)$ in $\mathbb{M}_{d, k}$ (called typical mark) with distribution $Q_{d, k}$ the sequence $\left(\left(\Theta_{i}, \Xi_{i}\right)\right)_{i \geq 1}$ of independent copies of $\left(\Theta_{0}, \Xi_{0}\right)$ is independent of the unmarked stationary Poisson point process $\Pi_{\lambda}=\sum_{i \geq 1} \delta_{X_{i}}$ on $\mathbb{R}^{d-k}$ with intensity $\lambda=\mathbf{E} \#\left\{i \geq 1: X_{i} \in\right.$ $\left.[0,1]^{d-k}\right\}$.

Note that $\left(\Theta_{0}, \Xi_{0}\right)$ specifies direction and base of the typical $k$-cylinder $\Theta_{0}\left(\left\{\left(\xi, \mathbf{o}_{k}\right)^{T}: \xi \in\right.\right.$ $\left.\Xi_{0}\right\} \oplus \mathbb{E}_{k}$ ) ( expressed in short form by $\Theta_{0}\left(\Xi_{0} \times \mathbb{R}^{k}\right)$ ) of the corresponding stationary Poisson $k$-cylinder process in $\mathbb{R}^{d}$ driven by $\Pi_{\lambda, Q_{d, k}}$ and defined by the countable family of random $k$-cylinders

$$
\left\{\Theta_{i}\left(\left(\Xi_{i}+X_{i}\right) \times \mathbb{R}^{k}\right)=\Theta_{i}\left(\left\{\left(\xi+X_{i}, \mathbf{o}_{k}\right)^{T}: \xi \in \Xi_{i}\right\} \oplus \mathbb{E}_{k}\right), i \geq 1\right\}
$$

In addition we assume that

$$
\mathbf{E} \nu_{d-k}\left(\Xi_{0} \oplus B_{\varepsilon}^{d-k}\right)<\infty
$$

for some $\varepsilon>0$, where $B_{\varepsilon}^{d-k}:=\left\{x \in \mathbb{R}^{d-k}:\|x\| \leq \varepsilon\right\}$ and $\nu_{d-k}$ denotes the Lebesgue measure on $\mathbb{R}^{d-k}$ for $k=0,1, \ldots, d$.

Finally, we are in a position to present the following

Definition 1.1: A stationary P- $k$-CM $\Xi_{\lambda, Q_{d, k}}$ in $\mathbb{R}^{d}$ is defined to be the countable union over the Poisson- $k$-cylinder process (1.1),

$$
\Xi_{\lambda, Q_{d, k}}:=\bigcup_{i \geq 1} \Theta_{i}\left(\left(\Xi_{i}+X_{i}\right) \times \mathbb{R}^{k}\right)
$$

provided that (1.2) is satisfied which ensures the $\mathbf{P}$-a.s. closedness of $\Xi_{\lambda, Q_{d, k}}$.

Remark 1.1: In other words, $\Xi_{\lambda, Q_{d, k}}$ can be considered as random variable taking values in the measurable space $\left[\mathcal{F}_{d}, \sigma_{f}\right]$, where $\sigma_{f}$ is the smallest $\sigma$-algebra containing all sets $\mathcal{F}_{C}:=$ $\left\{F \in \mathcal{F}_{d}: F \cap C \neq \emptyset\right\}$ for $C \in \mathcal{K}_{d}$, see [14] for details. The capacity or hitting functional of $\Xi_{\lambda, Q_{d, k}}$ is then given by 


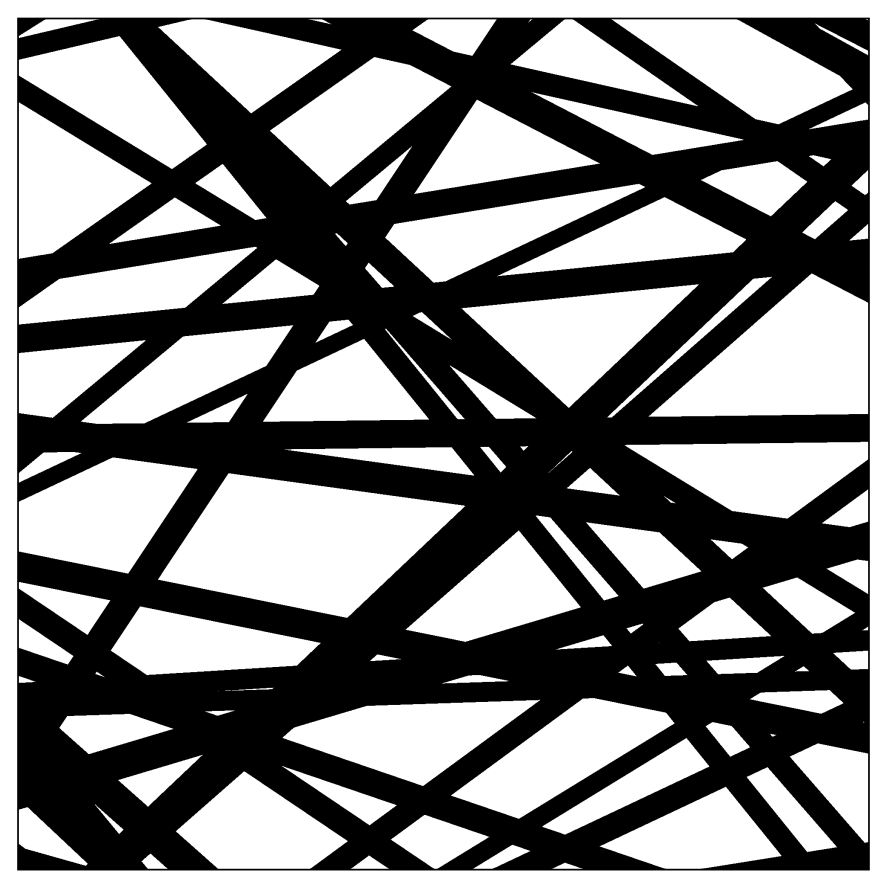

Figure 1: Realization of a planar stationary and isotropic Poisson 1-cylinder model

$$
T_{\lambda, Q_{d, k}}(C):=\mathbf{P}\left(\Xi_{\lambda, Q_{d, k}} \in \mathcal{F}_{C}\right)=1-\exp \left\{-\lambda \mathbf{E} \nu_{d-k}\left(\Xi_{0} \oplus \pi_{d-k}\left(-\Theta_{0}^{T} C\right)\right)\right\}
$$

for $C \in \mathcal{K}_{d}$, see [8], [10]. Here, $\pi_{d-k}(B):=\left\{\pi_{d-k}(x): x \in B\right\}$ for any $B \subset \mathbb{R}^{d}$ and $\pi_{d-k}(x)$ denotes the projection on the first $d-k$ components of $x \in \mathbb{R}^{d}$. Notice that the probability space $[\Omega, \sigma(\Omega), \mathbf{P}]$ can be chosen in such way that the indicator function $\mathbb{R}^{d} \times \Omega \ni(x, \omega) \mapsto$ $1\left(x \in \Xi_{\lambda, Q_{d, k}}(\omega)\right)$ is $\mathcal{B}_{d} \otimes \sigma(\Omega)$-measurable, see Appendix in [7] and [8].

\section{Remark 1.2:}

- The degenerate case $k=0\left(\mathbb{E}_{0}=\left\{\mathbf{o}_{d}\right\}\right.$ and $\left.\Theta_{0}=\mathrm{id}\right)$ yields the well-studied Boolean model, see e.g. [14], [3].

- In the special case $\Xi_{0}=\left\{\mathbf{o}_{d-k}\right\}$ the RACS $\Xi_{\lambda, Q_{d, k}}$ coincides with (the union of) a stationary Poisson $k$-flat process, see [14], [16], [19].

Next, we recall the notion of ergodicity and various mixing properties of RACSs, see [6], [10] and [19] for details. For this we need a family of shift operators $\left\{S_{x}: x \in \mathbb{R}^{d}\right\}$ defined by $S_{x} F:=\{y+x: y \in F\}$ for $F \in \mathcal{F}_{d}, S_{x} \mathcal{A}:=\left\{S_{x} F: F \in \mathcal{A}\right\}$ for $\mathcal{A} \in \sigma_{f}$ and a suitable family of sets growing unboundedly in all directions. 
Definition 1.2: (see [4], p. 196) A sequence of sets $\left(W_{n}\right)_{n \in \mathbb{N}}$ is called convex averaging sequence (short: CAS) in $\mathbb{R}^{d}$ if

1. $W_{n} \in \mathcal{C}_{d}$ and $W_{n} \subset W_{n+1}$ for each $n \in \mathbb{N}$,

2. $\varrho_{n}:=\sup \left\{r>0: B_{r}^{d}+x \subseteq W_{n}\right.$ for a $\left.x \in W_{n}\right\} \underset{n \rightarrow \infty}{\longrightarrow} \infty$.

It can be shown that $(2)$ is equivalent to $\nu_{d-1}\left(\partial W_{n}\right) / \nu_{d}\left(W_{n}\right) \underset{n \rightarrow \infty}{\longrightarrow} 0$, where $\nu_{d-1}\left(\partial W_{n}\right)$ denotes the surface content of $W_{n}$, see [9], p. 133.

Definition 1.2: A stationary RACS $\Xi$ in $\mathbb{R}^{d}$ with distribution $P_{\Xi}$ is said to be ergodic, weakly mixing resp. mixing if, for a $\mathrm{CAS}\left(W_{n}\right)_{n \in \mathbb{N}}$ and all $\mathcal{A}_{0}, \mathcal{A}_{1} \in \sigma_{f}$,

$$
\begin{array}{ll} 
& \frac{1}{\nu_{d}\left(W_{n}\right)} \int_{W_{n}} P_{\Xi}\left(\mathcal{A}_{0} \cap S_{x} \mathcal{A}_{1}\right) \mathrm{d} x \underset{n \rightarrow \infty}{\longrightarrow} P_{\Xi}\left(\mathcal{A}_{0}\right) P_{\Xi}\left(\mathcal{A}_{1}\right), \\
& \frac{1}{\nu_{d}\left(W_{n}\right)} \int_{W_{n}}\left|P_{\Xi}\left(\mathcal{A}_{0} \cap S_{x} \mathcal{A}_{1}\right)-P_{\Xi}\left(\mathcal{A}_{0}\right) P_{\Xi}\left(\mathcal{A}_{1}\right)\right| \mathrm{d} x \underset{n \rightarrow \infty}{\longrightarrow} 0 \\
\text { resp. } & P_{\Xi}\left(\mathcal{A}_{0} \cap S_{x} \mathcal{A}_{1}\right) \underset{\|x\| \rightarrow \infty}{\longrightarrow} P_{\Xi}\left(\mathcal{A}_{0}\right) P_{\Xi}\left(\mathcal{A}_{1}\right) .
\end{array}
$$

Furthermore, $\Xi$ is said to be mixing of $\operatorname{order} \ell(\geq 2)$ if for all $\mathcal{A}_{0}, \mathcal{A}_{1}, \ldots, \mathcal{A}_{\ell} \in \sigma_{f}$,

$$
P_{\Xi}\left(\mathcal{A}_{0} \cap S_{x_{n, 1}} \mathcal{A}_{1} \cap \cdots \cap S_{x_{n, \ell}} \mathcal{A}_{\ell}\right) \underset{n \rightarrow \infty}{\longrightarrow} P_{\Xi}\left(\mathcal{A}_{0}\right) P_{\Xi}\left(\mathcal{A}_{1}\right) \cdots P_{\Xi}\left(\mathcal{A}_{k}\right)
$$

as $\left\|x_{n, i}\right\|_{n \rightarrow \infty} \infty$ for $i=1, \ldots, \ell$ in such a way that $\left\|x_{n, i}-x_{n, j}\right\|_{n \rightarrow \infty} \infty$ also for all $i \neq j$, see [4] (p. 215) for $\ell$ th order mixing of random (counting) measures.

Obviously, mixing of order $\ell(\geq 2) \Longrightarrow$ mixing $\Longrightarrow$ weak mixing $\Longrightarrow$ ergodic but the reverse implications do not hold in general.

Remark 1.3: In view of Lemma 4 in [6] the sets $\mathcal{A}_{0}, \mathcal{A}_{1}$ in the limits (1.5) - (1.7) can be replaced by $\mathcal{F}^{C_{i}}:=\left\{F \in \mathcal{F}_{d}: F \cap C_{i}=\emptyset\right\}$ for $i=0,1$ and all $C_{0}, C_{1} \in \mathcal{K}_{d}$. In the same way the condition (1.8) can be reformulated with $\mathcal{F}^{C_{i}}$ for $C_{i} \in \mathcal{K}_{d}$ instead of $\mathcal{A}_{i}$ for $i=0,1, \ldots, \ell$.

\section{Main Results on Mixing of Poisson k-Cylinder Models}

Since a stationary P-0-CM can be identified with a stationary Boolean model which is always mixing ( of any order), see [19] or [6], we only need to consider P- $k$-CMs for $k=1, \ldots, d-1$.

Theorem 2.1. For each $k=1, \ldots, d-1$, the stationary P-k-CM (1.3) satisfying (1.2) is weakly mixing (and thus also ergodic). 
Proof. Let $P_{\lambda, Q_{d, k}}$ denote the distribution of the RACS $\Xi_{\lambda, Q_{d, k}}$. According to Remark 1.3 we need to prove (1.6) only for $\mathcal{A}_{i}=\mathcal{F}^{C_{i}}, i=0,1$. Since $\mathcal{F}^{C_{0}} \cap S_{x} \mathcal{F}^{C_{1}}=\mathcal{F}^{C_{0} \cup S_{x} C_{1}}$ and the relation $P_{\lambda, Q_{d, k}}\left(\mathcal{F}^{C}\right)=1-T_{\lambda, Q_{d, k}}(C)$ for any $C \in \mathcal{K}_{d}$, which follows from (1.4), we shall show the limit

$$
\lim _{n \rightarrow \infty} \frac{1}{\nu_{d}\left(W_{n}\right)} \int_{W_{n}}\left|1-T_{\lambda, Q_{d, k}}\left(C_{0} \cup S_{x} C_{1}\right)-\left(1-T_{\lambda, Q_{d, k}}\left(C_{0}\right)\right)\left(1-T_{\lambda, Q_{d, k}}\left(C_{1}\right)\right)\right| \mathrm{d} x=0
$$

for all $C_{0}, C_{1} \in \mathcal{K}_{d}$, where $\left(W_{n}\right)_{n \in \mathbb{N}}$ is an arbitrary CAS in $\mathbb{R}^{d}$. For notational ease we use here and throughout Section 2 the abbreviations

$$
\widetilde{K}_{i}:=K \oplus \pi_{d-k}\left(-\theta^{T} C_{i}\right) \quad \text { for all } \quad(\theta, K) \in \mathbb{M}_{d, k} \quad \text { or } \quad \widetilde{\Xi}_{i}:=\Xi_{0} \oplus \pi_{d-k}\left(-\Theta_{0}^{T} C_{i}\right)
$$

for all $i=0,1$. An application of formula (1.4) expressing the capacity functional of $\Xi_{\lambda, Q_{d, k}}$ in combination with the identity

$$
\begin{aligned}
& \nu_{d-k}\left(K \oplus \pi_{d-k}\left(-\theta^{T}\left(C_{0} \cup S_{x} C_{1}\right)\right)\right)=\nu_{d-k}\left(\widetilde{K}_{0} \cup\left(\widetilde{K}_{1}-\pi_{d-k}\left(\theta^{T} x\right)\right)\right) \\
& =\nu_{d-k}\left(\widetilde{K}_{0}\right)+\nu_{d-k}\left(\widetilde{K}_{1}\right)-\nu_{d-k}\left(\widetilde{K}_{0} \cap\left(\widetilde{K}_{1}-\pi_{d-k}\left(\theta^{T} x\right)\right)\right)
\end{aligned}
$$

reveals that the previous limiting relation is equivalent to

$$
R_{n}:=\frac{1}{\nu_{d}\left(W_{n}\right)} \int_{W_{n}}\left(\exp \left\{\lambda \mathbf{E} \nu_{d-k}\left(\widetilde{\Xi}_{0} \cap\left(\widetilde{\Xi}_{1}-\pi_{d-k}\left(\Theta_{0}^{T} x\right)\right)\right)\right\}-1\right) \mathrm{d} x \underset{n \rightarrow \infty}{\longrightarrow} 0 .
$$

The elementary inequality $e^{y}-1 \leq y e^{y}$ for $y \geq 0$ and

$$
\mathbf{E} \nu_{d-k}\left(\widetilde{\Xi}_{0} \cap\left(\widetilde{\Xi}_{1}-\pi_{d-k}\left(\Theta_{0}^{T} x\right)\right)\right) \leq \gamma:=\min \left\{\mathbf{E} \nu_{d-k}\left(\widetilde{\Xi}_{0}\right), \mathbf{E} \nu_{d-k}\left(\widetilde{\Xi}_{1}\right)\right\}<\infty
$$

yield the estimate

$$
R_{n} \leq \frac{\lambda e^{\lambda \gamma}}{\nu_{d}\left(W_{n}\right)} \int_{W_{n}} \mathbf{E} \nu_{d-k}\left(\widetilde{\Xi}_{0} \cap\left(\widetilde{\Xi}_{1}-\pi_{d-k}\left(\Theta_{0}^{T} x\right)\right)\right) \mathrm{d} x=\lambda e^{\lambda \gamma} \mathbf{E} \widetilde{R}_{n}\left(\Theta_{0}, \Xi_{0}\right)
$$

where

$$
\widetilde{R}_{n}(\theta, K)=\frac{1}{\nu_{d}\left(W_{n}\right)} \int_{\theta^{T} W_{n}} \nu_{d-k}\left(\widetilde{K}_{0} \cap\left(\widetilde{K}_{1}-\pi_{d-k}(x)\right)\right) \mathrm{d} x \quad \text { for } \quad(\theta, K) \in \mathbb{M}_{d, k} .
$$

It is easily seen that $\widetilde{R}_{n}(\theta, K)$ is bounded by $\min \left\{\nu_{d-k}\left(\widetilde{K}_{0}\right), \nu_{d-k}\left(\widetilde{K}_{1}\right)\right\}$ for all $(\theta, K) \in \mathrm{M}_{d, k}$ and this bound is integrable with respect to $Q_{d, k}$. Thus, in order to obtain (2.2) via Lebesgue's dominated convergence theorem it remains to show $\widetilde{R}_{n}(\theta, K) \underset{n \rightarrow \infty}{\longrightarrow} 0$ for any fixed $(\theta, K) \in \mathbb{M}_{d, k}$. 
Since the support of the function $\mathbb{R}^{d} \ni x \mapsto \nu_{d}\left(\widetilde{K}_{0} \cap\left(\widetilde{K}_{1}-\pi_{d-k}(x)\right)\right)$ is unbounded, we split $\mathbb{R}^{d}$ into the orthogonal subspaces $\mathbb{E}_{k}^{\perp}$ and $\mathbb{E}_{k}$. For this purpose, let $\nu_{\mathbb{L}}$ denote the Lebesgue measure on an affine subspace $\mathbb{L}$ of $\mathbb{R}^{d}$ which can be identified with $\nu_{p}$ if $p=\operatorname{dim} \mathbb{L}$. By applying Fubini's theorem we obtain that

$$
\begin{aligned}
\widetilde{R}_{n}(\theta, K) & =\int_{\mathbb{E}_{k}^{\perp}} \int_{\mathbb{E}_{k}} \frac{1_{\theta^{T} W_{n}}(y+z)}{\nu_{d}\left(W_{n}\right)} \nu_{d-k}\left(\widetilde{K}_{0} \cap\left(\widetilde{K}_{1}-\pi_{d-k}(y+z)\right)\right) \nu_{\mathbb{E}_{k}}(\mathrm{~d} z) \nu_{\mathbb{E}_{k}^{\perp}}(\mathrm{d} y) \\
& =\int_{\mathbb{E}_{k}^{\perp}} \frac{\nu_{\mathbb{E}_{k}}\left(\left(\theta^{T} W_{n}-y\right) \cap \mathbb{E}_{k}\right)}{\nu_{d}\left(W_{n}\right)} \nu_{d-k}\left(\widetilde{K}_{0} \cap\left(\widetilde{K}_{1}-y\right)\right) \nu_{\mathbb{E}_{k}^{\perp}}(\mathrm{d} y) .
\end{aligned}
$$

Although it seems to be intuitively clear that $\nu_{\mathbb{E}_{k}}\left(\left(\theta^{T} W_{n}-y\right) \cap \mathbb{E}_{k}\right) / \nu_{d}\left(\theta^{T} W_{n}\right) \longrightarrow 0$ as $n \rightarrow \infty$, we give a rigouros reasoning for this by employing the following result proved in [15]: For any $C \in \mathcal{C}_{d}$ and affine subspaces $\mathbb{L}_{1}, \ldots, \mathbb{L}_{m}$ of $\mathbb{R}^{d}$ with $\operatorname{dim} \mathbb{L}_{j}=d_{j} \geq 1$ such that $d_{1}+\cdots+d_{m}=d$, the inequality

$$
\nu_{d}(C) \geq \frac{d_{1} ! \cdots d_{m} !}{d !} \nu_{d_{1}}\left(C \cap \mathbb{L}_{1}\right) \cdots \nu_{d_{m}}\left(C \cap \mathbb{L}_{m}\right)
$$

holds so that

$$
\frac{\nu_{E_{k}}\left(\left(\theta^{T} W_{n}-y\right) \cap \mathbb{E}_{k}\right)}{\nu_{d}\left(\theta^{T} W_{n}\right)} \leq \frac{\left(\begin{array}{c}
d \\
k
\end{array}\right)}{\nu_{\mathbb{E}_{k}^{\perp}}\left(\left(\theta^{T} W_{n}-y\right) \cap \mathbb{E}_{k}^{\perp}\right)}=\frac{\left(\begin{array}{c}
d \\
k
\end{array}\right)}{\nu_{d-k}\left(\theta^{T} W_{n} \cap \mathbb{E}_{k}^{\perp}\right)}
$$

for all $y \in \mathbb{E}_{k}^{\perp}$ and $\theta \in \mathbb{S O}_{d, k}$. Since $\theta^{T} W_{n}$ is a CAS in $\mathbb{R}^{d}$ it follows from Definition 1.2 that

$$
\nu_{d-k}\left(\theta^{T} W_{n} \cap \mathbb{E}_{k}^{\perp}\right) \geq \nu_{d-k}\left(B_{\varrho_{n}}^{d-k}\right) \longrightarrow \infty \quad \text { as } \quad n \rightarrow \infty .
$$

Finally, together with

$$
\int_{\mathbb{E}_{k}^{\perp}} \nu_{d-k}\left(\widetilde{K}_{0} \cap\left(\widetilde{K}_{1}-y\right)\right) \nu_{E_{k}^{\perp}}(\mathrm{d} y)=\nu_{d-k}\left(\widetilde{K}_{0}\right) \nu_{d-k}\left(\widetilde{K}_{1}\right)
$$

we arrive at

$$
\widetilde{R}_{n}(\theta, K) \leq\left(\begin{array}{l}
d \\
k
\end{array}\right) \frac{\nu_{d-k}\left(\widetilde{K}_{1}\right) \nu_{d-k}\left(\widetilde{K}_{2}\right)}{\nu_{d-k}\left(B_{\varrho_{n}}^{d-k}\right)} \underset{n \rightarrow \infty}{\longrightarrow} 0 .
$$

This completes the proof of Theorem 2.1.

It is well-know, see Theorem 10.5.3 in [19], that a stationary Poisson hyperplane process (= $\mathrm{P}-(d-1)$-CM with $\left.\Xi_{0}=\left\{\mathbf{o}_{d-1}\right\}\right)$ is mixing if its spherical directional distribution (defined on $\left.S^{d-1}\right)$ vanishes on every great subsphere $S^{d-1} \cap \mathbb{L}$ for $\mathbb{L} \in \mathcal{G}(d, d-1)$. A corresponding generalization of this result for any stationary $\mathrm{P}-k$-CMs is given in the following 
Theorem 2.2. For each $k=1, \ldots, d-1$, the stationary $P-k-C M$ (1.3) satisfying (1.2) is mixing if and only if the directional distribution $Q_{d, k}^{(0)}(\cdot):=Q_{d, k}\left(\cdot \times \mathcal{K}_{d-k}^{\prime}\right)$ fulfills the condition

$$
Q_{d, k}^{(0)}\left(\left\{\theta \in \mathbb{S O}_{d, k}: u \in \theta \mathbb{E}_{k}\right\}\right)=0 \quad \text { for all } u \in S^{d-1}
$$

Proof. We use the notation introduced in the proof of Theorem 2.1. Taking into account Remark 1.3, the shape of the capacity functional (1.4), the decomposition (2.1), and (2.2) we recognize that $\Xi_{\lambda, Q_{d, k}}$ is mixing if and only if

$$
\exp \left\{\lambda \mathbf{E} \nu_{d-k}\left(\widetilde{\Xi}_{0} \cap\left(\widetilde{\Xi}_{1}-\pi_{d-k}\left(\Theta_{0}^{T} x_{n}\right)\right)\right)\right\}-1 \underset{n \rightarrow \infty}{\longrightarrow} 0 \quad \text { for all } \quad C_{0}, C_{1} \in \mathcal{K}_{d}
$$

and any sequence $\left(x_{n}\right)_{n \in \mathbb{N}} \subset \mathbb{R}^{d}$ satisfying $\left\|x_{n}\right\| \underset{n \rightarrow \infty}{\longrightarrow} \infty$, or equivalently

$$
\lim _{n \rightarrow \infty} \int_{\mathbb{M}_{d, k}} \nu_{d-k}\left(\widetilde{K}_{0} \cap\left(\widetilde{K}_{1}-\pi_{d-k}\left(\theta^{T} x_{n}\right)\right)\right) Q_{d, k}(\mathrm{~d}(\theta, K))=0 .
$$

Let us first show that (2.4) implies (2.5). By (2.3) and Lebesgue's dominated convergence theorem it suffices to show that, for any fixed $K \in \mathcal{K}_{d-k}^{\prime}$ and $C_{0}, C_{1} \in \mathcal{K}_{d}$

$$
\lim _{n \rightarrow \infty} \nu_{d-k}\left(\widetilde{K}_{0} \cap\left(\widetilde{K}_{1}-\pi_{d-k}\left(\theta^{T} x_{n}\right)\right)\right)=0 \quad \text { for } \quad Q_{d, k}^{(0)}-\text { almost all } \quad \theta \in \mathbb{S O}_{d, k}
$$

Obviously, due to $\widetilde{K}_{0}, \widetilde{K}_{1} \in \mathcal{K}_{d-k}^{\prime},(2.6)$ holds true if $\left\|\pi_{d-k}\left(\theta^{T} x_{n}\right)\right\| \rightarrow \infty$ as $n \rightarrow \infty$ for $Q_{d, k^{-}}^{(0)}$ almost all $\theta \in \mathbb{S O}_{d, k}$. Suppose there is some Borel set $B \subset \mathbb{S O}_{d, k}$ such that $Q_{d, k}^{(0)}(B)>0$ and $\liminf _{n \rightarrow \infty}\left\|\pi_{d-k}\left(\theta^{T} x_{n}\right)\right\|<\infty$ for $\theta \in B$. Thus, putting $u_{n}:=x_{n} /\left\|x_{n}\right\| \in S^{d-1}$ it follows together with $\pi_{d-k}\left(\theta^{T} u_{n}\right)=\pi_{d-k}\left(\theta^{T} x_{n}\right) /\left\|x_{n}\right\|$ that $\liminf _{n \rightarrow \infty}\left\|\pi_{d-k}\left(\theta^{T} u_{n}\right)\right\|=0$ for $\theta \in B$. Since $S^{d-1} \in \mathcal{K}_{d}^{\prime}$ there exists a subsequence $\left(u_{n_{m}}\right)_{m \in \mathbb{N}}$ having the limit $u \in S^{d-1}$ as $m \rightarrow \infty$ satisfying $\pi_{d-k}\left(\theta^{T} u\right)=\mathbf{o}_{d-k}$ (i.e. $u \in \theta \mathbb{E}_{k}$ ) for $\theta \in B$. But this is a contradiction to condition (2.4). Hence, (2.4) implies the mixing property of the RACS $\Xi_{\lambda, Q_{d, k}}$.

To prove the reverse direction we assume the contrary of (2.4), i.e. there exists an $u_{0} \in S^{d-1}$ such that $Q_{d, k}^{(0)}\left(\left\{\theta \in \mathbb{S O}_{d, k}: \pi_{d-k}\left(\theta^{T} u_{0}\right)=\mathbf{o}_{d-k}\right\}\right)=\varepsilon>0$. Choosing $C_{0}=C_{1}=B_{1}^{d}$ and $x_{n}=n u_{0}$ for all $n \in \mathbb{N}$ we conclude that

$$
\begin{aligned}
& \int_{\mathbb{M}_{d, k}} \nu_{d-k}\left(\widetilde{K}_{0} \cap\left(\widetilde{K}_{1}-n \pi_{d-k}\left(\theta^{T} u_{0}\right)\right)\right) Q_{d, k}(\mathrm{~d}(\theta, K)) \\
\geq & \int_{\left\{\theta: \pi_{d-k}\left(\theta^{T} u_{0}\right)=\mathbf{o}_{d-k}\right\} \times \mathcal{K}_{d-k}^{\prime}} \nu_{d-k}\left(\widetilde{K}_{0} \cap\left(\widetilde{K}_{1}-n \pi_{d-k}\left(\theta^{T} u_{0}\right)\right)\right) Q_{d, k}(\mathrm{~d}(\theta, K)) \\
= & \int_{\left\{\theta: \pi_{d-k}\left(\theta^{T} u_{0}\right)=\mathbf{o}_{d-k}\right\} \times \mathcal{K}_{d-k}^{\prime}} \nu_{d-k}\left(K \oplus B_{1}^{d-k}\right) Q_{d, k}(\mathrm{~d}(\theta, K)) \\
\geq & \varepsilon \nu_{d-k}\left(B_{1}^{d-k}\right)>0 \text { for all } n \in \mathbb{N} .
\end{aligned}
$$


But this means that (2.5) does not hold and thus the P-k-CM $\Xi_{\lambda, Q_{d, k}}$ is not mixing. In other words, (2.4) is necessary to ensure the mixing property (1.7) for $\Xi_{\lambda, Q_{d, k}}$. This completes the proof of Theorem 2.2 .

Theorem 2.3. For each $1 \leq k \leq d-1$, the stationary P-k-CM (1.3) satisfying (1.2) and the condition (2.4) is mixing of any order $\ell \geq 2$.

Proof. First, we rewrite (1.8) according to Remark 1.3 in terms of the hitting functional $T_{\lambda, Q_{d, k}}(C)=1-\exp \{-\mu(C)\}$ with $\mu(C):=\lambda \mathbf{E} \nu_{d-k}\left(\Xi_{0} \oplus \pi_{d-k}\left(-\Theta_{0}^{T} C\right)\right)$. This means we need to prove that, for any $C_{0}, C_{1}, \ldots C_{\ell} \in \mathcal{K}_{d}$ and sequences $x_{n, 0} \equiv \mathbf{o}_{d}, x_{n, 1}, \ldots, x_{n, \ell}$ satisfying $\left\|x_{n, i}-x_{n, j}\right\| \underset{n \rightarrow \infty}{\longrightarrow} \infty$ for $0 \leq i<j \leq \ell$,

$$
\Delta_{n}\left(C_{0}, \ldots, C_{\ell}\right):=1-T_{\lambda, Q_{d, k}}\left(\bigcup_{i=0}^{\ell} S_{x_{n, i}} C_{i}\right)-\prod_{i=0}^{\ell}\left(1-T_{\lambda, Q_{d, k}}\left(C_{i}\right)\right) \underset{n \rightarrow \infty}{\longrightarrow} 0 .
$$

It is easily seen that $\Delta_{n}\left(C_{0}, \ldots, C_{\ell}\right) \geq 0$ and

$$
\begin{aligned}
\Delta_{n}\left(C_{0}, \ldots, C_{\ell}\right) & =\exp \left\{-\mu\left(\bigcup_{i=0}^{\ell} S_{x_{n, i}} C_{i}\right)\right\}-\exp \left\{-\sum_{i=0}^{\ell} \mu\left(C_{i}\right)\right\} \\
& \leq \exp \left\{\sum_{i=0}^{\ell} \mu\left(C_{i}\right)-\mu\left(\bigcup_{i=0}^{\ell} S_{x_{n, i}} C_{i}\right)\right\}-1 \\
& \leq \exp \left\{\sum_{0 \leq i<j \leq \ell} \lambda \mathbf{E} \nu_{d-k}\left(\widetilde{\Xi}_{i} \cap\left(\widetilde{\Xi}_{j}-\pi_{d-k}\left(\Theta_{0}\left(x_{n, j}-x_{n, i}\right)\right)\right)\right)\right\}-1,
\end{aligned}
$$

where $\widetilde{\Xi}_{j}:=\Xi_{0} \oplus \pi_{d-k}\left(-\Theta_{0}^{T} C_{j}\right)$ for $j=0,1, \ldots, \ell$. The last bound results from the additivity of the Lebesgue measure $\nu_{d-k}$ combined with its translation-invariance yielding, among others, $\mu\left(S_{x_{n, i}} C_{i}\right)=\mu\left(C_{i}\right)$. Finally, repeating the proof of (2.5) leads to the limits

$$
\mathbf{E} \nu_{d-k}\left(\widetilde{\Xi}_{i} \cap\left(\widetilde{\Xi}_{j}-\pi_{d-k}\left(\Theta_{0}^{T}\left(x_{n, j}-x_{n, i}\right)\right)\right) \underset{n \rightarrow \infty}{\longrightarrow} 0 \quad \text { if } \quad\left\|x_{n, i}-x_{n, j}\right\|_{n \rightarrow \infty}^{\longrightarrow} \infty\right.
$$

for $0 \leq i<j \leq \ell$. Thus, $\Delta_{n}\left(C_{0}, \ldots, C_{\ell}\right) \underset{n \rightarrow \infty}{\longrightarrow} 0$ for any $\ell \geq 2$ which provides the assertion of Theorem 2.3 .

Remark 2.1: The shape of the hitting functional (1.4) with $\mu(C) \in[0, \infty)$ (being a completely alternating semicontinuous capacity on $\mathcal{K}_{d}$ such that $\mu(\emptyset)=0$ ) reveals that every P- $k$-CM $\Xi_{\lambda, Q_{d, k}}$ (satisfying (1.2)) is an union-infinite divisible stationary RACS in $\mathbb{R}^{d}$ without fixed points, see Theorem 2.3.3 in [19] and Chapt. 4.1 in [18]. 
Corollary 2.4. For each $k=1, \ldots, d-1$, the P-k-CM $\Xi_{\lambda, Q_{d, k}}$ is not mixing if the directional distribution $Q_{d, k}^{(0)}$ has atoms.

Proof. Let $Q_{d, k}^{(0)}\left(\left\{\vartheta_{0}\right\}\right)>0$ for some $\vartheta_{0} \in \mathbb{S O}_{d, k}$. Then $Q_{d, k}^{(0)}\left(\left\{\theta \in \mathbb{S O}_{d, k}: u \in \theta \mathbb{E}_{k}\right\}\right) \geq$ $Q_{d, k}^{(0)}\left(\left\{\vartheta_{0}\right\}\right)>0$ for all $u \in S^{d-1} \cap \vartheta_{0} \mathbb{E}_{k}$.

Now, let $\mu_{d, k}$ denote the restriction of the unique normalized rotation invariant (Haar) measure $\mu_{d}$ on $\mathbb{S O}_{d}$, see Chapt. 13.2 in [19], to $\mathbb{S O}_{d, k}$. Two linear subspaces $\mathbb{L}$ and $\mathbb{L}^{\prime}$ of $\mathbb{R}^{d}$ are said to be in special position (in general position otherwise) if

$$
\operatorname{span}\left(\mathbb{L} \cup \mathbb{L}^{\prime}\right) \neq \mathbb{R}^{d} \quad \text { and } \quad \operatorname{dim}\left(\mathbb{L} \cap \mathbb{L}^{\prime}\right)>0 .
$$

Corollary 2.5. For each $k=1, \ldots, d-1$, the stationary P-k-CM (1.3) satisfying (1.2) is mixing iff

$$
Q_{d, k}^{(0)}\left(\left\{\theta \in \mathbb{S O}_{d, k}: \theta \mathbb{E}_{k} \text { and } \mathbb{L} \text { are in special position }\right\}\right)=0 \text { for all } \mathbb{L} \in \mathcal{G}(d, 1) \text {. }
$$

In particular $\Xi_{\lambda, Q_{d, k}}$ is mixing if $Q_{d, k}^{(0)}$ is absolute continuous w.r.t. $\mu_{d, k}$.

Proof. It is easily seen that, for all $u \in S^{d-1}$ and $\theta \in \mathbb{S O}_{d, k}$,

$$
u \in \theta \mathbb{E}_{k} \quad \text { iff } \quad \operatorname{span}(u) \text { and } \theta \mathbb{E}_{k} \quad \text { are in special position } .
$$

On the other hand, from Lemma 13.1.2 in [19] we know that $\mu_{d}\left(\left\{\theta \in \mathbb{S O}_{d}: \theta \mathbb{E}_{k}\right.\right.$ and $\mathbb{L}$ are in special position $\left.\}\right)=0$.

In general, condition (2.4) turns out to be stronger than $Q_{d, k}^{(0)}(\{\theta\})=0$ for all $\theta \in \mathbb{S O}_{d, k}$. However, in the particular case $d=2, k=1$ both conditions are equivalent.

Example: Let $Q_{0}^{(d)}$ denote the image measure of $Q_{d, d-1}^{(0)}$ under the mapping $\mathbb{S O}_{d} \ni \theta \mapsto \theta e_{1} \in S^{d-1}$. Then $Q_{0}^{(d)}$ is a probability measure on the sphere $S^{d-1}$ and condition (2.4) can be expressed as

$$
Q_{0}^{(d)}\left(S^{d-1} \cap \mathbb{L}\right)=0 \quad \text { for all } \quad \mathbb{L} \in \mathcal{G}(d, d-1)
$$

confirming once more the above-mentioned result in [19], p. 517.

To study weak dependence properties of a stationary RACS $\Xi$ in $\mathbb{R}^{d}$ which go beyond mixing, see e.g. [12], [13] in case of STIT tessellations, we consider the tail- $\sigma$-algebra $\sigma_{f}^{\infty}(\Xi):=$ 
$\bigcap_{n \in \mathbb{N}} \sigma_{f}\left(\Xi \cap\left\{x \in \mathbb{R}^{d}:\|x\| \geq n\right\}\right)$, where $\sigma_{f}\left(\Xi^{\prime}\right)$ is the smallest $\sigma$-algebra containing all events $\left\{\Xi^{\prime} \in \mathcal{F}_{C}\right\}=\left\{\Xi^{\prime} \cap C \neq \emptyset\right\}$ for $C \in \mathcal{K}_{d}$.

It is a well-known fact, see [4] for stationary point processes, that the triviality of the tail$\sigma$-algebra $\sigma_{f}^{\infty}(\Xi)$, i.e. $\mathbf{P}(A) \in\{0,1\}$ for all tail events $A$, implies that $\Xi$ is mixing (even of any order). On the other hand, the reverse implication is false in general. Following the terminology in [11], a stationary RACS $\Xi$ in $\mathbb{R}^{d}$ having (non-)trivial tail- $\sigma$-algebra $\sigma_{f}^{\infty}(\Xi)$ is said to have (long) short range correlations or (long) short range dependences.

Remark 2.2: For each $k=1, \ldots, d-1$, the stationary $\mathrm{P}-k$-CM $\Xi_{\lambda, Q_{d, k}}$ has long range correlations. It is easily checked (and already mentioned in [10]) that the events $A_{\varepsilon}:=$ $\left\{\Xi_{\lambda, Q_{d, k}} \cap B_{\varepsilon}^{d}=\emptyset\right\}$ belong to $\sigma_{f}\left(\Xi_{\lambda, Q_{d, k}} \cap\left\{x \in \mathbb{R}^{d}:\|x\| \geq n\right\}\right)$ for all $n \in \mathbb{N}$ and $\varepsilon>0$, but $\mathbf{P}\left(A_{\varepsilon}\right)=1-T_{\lambda, Q_{d, k}}\left(B_{\varepsilon}^{d}\right) \in(0,1)$.

\section{A Remarkable Property of Cells Generated by a P-(d-1)-CM}

Throughout, in this section we consider exclusively $\mathbf{P}$ - $(d-1)$-CMs satisfying $\mathbf{P}\left(\Theta_{0} e_{1} \in \mathbb{L}\right)=$ $Q_{0}^{(d)}\left(S^{d-1} \cap \mathbb{L}\right)<1$ for all $\mathbb{L} \in \mathcal{G}(d, d-1$ ) (in particular if (2.7) holds) with typical base $\Xi_{0} \in \mathcal{C}_{1}$ satisfying (1.2) for $k=d-1$, i.e. $\Xi_{0}$ is a closed interval with finite mean length $\mathbf{E} \nu_{1}\left(\Xi_{0}\right)$ so that the $(d-1)$-cylinders can be regarded as randomly dilated hyperplanes in $\mathbb{R}^{d}$ and the complement of their union $\Xi_{\lambda, Q_{d, d-1}}^{c}$ consists of pairwise disjoint open bounded convex polytopes. By taking the closure of each of these open polytopes we we obtain a family $\left\{Z_{i}, i \geq 1\right\}$ of random compact convex polytopes satisfying $Z_{i} \cap Z_{j}=\emptyset$ or $\nu_{d}\left(Z_{i} \cap Z_{j}\right)=0$ otherwise for all $i \neq j$. Let $\mathcal{P}_{d}^{\prime}$ denote the subset of non-empty polytopes in $\mathcal{C}_{d}$.

To start with, we derive a formula for the contact distribution function $0 \leq r \mapsto H_{S}(r)$ of $\Xi:=\Xi_{\lambda, Q_{d, d-1}}$, see e.g. [3],

$$
H_{S}(r):=\mathbf{P}\left(\Xi \cap r S \neq \emptyset \mid \mathbf{o}_{d} \notin \Xi\right)=1-\frac{1-\mathbf{P}\left(\mathbf{o}_{d} \in \Xi \oplus(-r S)\right)}{1-\mathbf{P}\left(\mathbf{o}_{d} \in \Xi\right)}
$$

where the "structuring element" $S \in \mathcal{K}_{d}^{\prime}$ is star-shaped w.r.t. $\mathbf{o}_{d} \in S$. Straightforward calculations carried out in [8] and [10], see also [20] for a different approach, yield that $p(r):=\mathbf{P}\left(\mathbf{o}_{d} \in \Xi \oplus(-r S)\right)=1-\exp \left\{-\lambda \mathbf{E} \nu_{1}\left(\Xi_{0} \oplus r \pi_{1}\left(-\Theta_{0}^{T} S\right)\right)\right\}$ and the expression $p(0)=\mathbf{E} \nu_{d}\left(\Xi \cap[0,1]^{d}\right)=1-\exp \left\{-\lambda \mathbf{E} \nu_{1}\left(\Xi_{0}\right)\right\}$ for the volume fraction of $\Xi$. Inserting these formulas in (3.1) and taking into account that $\pi_{1}\left(-\Theta_{0}^{T} S\right)$ is an intervall we arrive at $H_{C}(r)=1-\exp \left\{-r \lambda \mathbf{E} \nu_{1}\left(\pi_{1}\left(-\Theta_{0}^{T} S\right)\right)\right\}$ for $r \geq 0$ which shows an exponential distribution function being always the same regardless of how $\nu_{1}\left(\Xi_{0}\right)$ is distributed. This interesting observation proves useful in the statistical analysis of $\Xi_{\lambda, Q_{d, d-1}}$ and is the consequence of an 
invariance property of the so-called zero cell $Z_{\mathbf{o}}$ which coincides with the unique polytope $Z_{i}$ whose interior $\operatorname{int}\left(Z_{i}\right)$ contains the origin $\mathbf{o}_{d}$ conditional on $\mathbf{o}_{d} \notin \Xi_{\lambda, Q_{d, d-1}}$.

A simple statistical application is the following: Let $\Xi$ be observed in a CAS $W_{n}$, see Definition 1.2. Then $\widehat{p}_{n}(r):=\nu_{d}\left(W_{n} \cap \Xi \oplus(-r S)\right) / \nu_{d}\left(W_{n}\right)$ is unbiased and strongly consistent estimator for $p(r)$, where the consistency results from Theorem 2.1 and the spatial ergodic theorem, see Chapt. 12.2 in [4]. Hence, the empirical contact distribution function $\widehat{H}_{S, n}(r)$ turns out to be strongly consistent (even uniformly),

$$
\widehat{H}_{S, n}(r):=1-\frac{1-\widehat{p}_{n}(r)}{1-\widehat{p}_{n}(0)} \underset{n \rightarrow \infty}{\stackrel{\mathbf{P}-\text { a.s. }}{\longrightarrow}} H_{S}(r) \quad \text { for } \quad r \geq 0
$$

such that, for $S=B_{1}^{d}$ and $r>0, \widehat{\lambda}_{n}:=-\log \left(1-\widehat{H}_{S, n}(r)\right) / 2 r \underset{n \rightarrow \infty}{\stackrel{\mathbf{P}-\text { a.s. }}{\longrightarrow}} \lambda$.

The above-mentioned invariance property was already mentioned in [16] and [17]. But neither there nor elsewhere - to the best of authors' knowledge - this rather surprising property of the stationary particle process $\left\{Z_{i}, i \geq 1\right\}$ has been precisely formulated and rigorously proved.

The family $\left\{Z_{i}, i \geq 1\right\}$ can be regarded as a stationary tessellation / mosaic, see Chapt. 10 in [19], with "thick boundaries". In Figure 1 the white polygons coincide with the interior of the closed cells $Z_{i}$ and the black strips form their boundaries. In accordance with the above definition the zero cell $Z_{\mathbf{o}}$ is a random element in $\mathcal{P}_{d}^{\prime}$ with (conditional) distribution

$$
P_{\mathbf{o}}(\mathcal{A}):=\frac{P_{\mathbf{o}}^{*}\left(\mathcal{A} \cap\left\{F \in \mathcal{F}_{d}: \mathbf{o}_{d} \in F\right\}\right)}{P_{\mathbf{o}}^{*}\left(\left\{F \in \mathcal{F}_{d}: \mathbf{o}_{d} \in F\right\}\right)} \quad \text { for } \quad \mathcal{A} \in \sigma_{f} \cap \mathcal{P}_{d}^{\prime}
$$

where $P_{\mathbf{o}}^{*}$ denotes the distribution of the random compact convex polytope

$$
Z_{\mathbf{o}}^{*}:=\left\{\begin{array}{lll}
\bigcup_{i \geq 1} 1\left(\mathbf{o}_{d} \in \operatorname{int}\left(Z_{i}\right)\right) Z_{i} & \text { if } & \mathbf{o}_{d} \notin \Xi_{\lambda, Q_{d, d-1}} \\
\emptyset & \text { if } & \mathbf{o}_{d} \in \Xi_{\lambda, Q_{d, d-1}}
\end{array}\right.
$$

On the other hand, the typical cell $\widehat{Z}_{\mathbf{o}}$ associated with the tessellation $\left\{Z_{i}, i \geq 1\right\}$ is defined via the Palm mark distribution $\widehat{P}_{\mathbf{o}}$ of the stationary marked point process $\Psi_{\alpha}:=\sum_{i \geq 1} \delta_{\left[\alpha\left(Z_{i}\right), Z_{i}-\alpha\left(Z_{i}\right)\right]}$ on $\mathbb{R}^{d}$ with measurable mark space $\left[\mathcal{P}_{d}^{\prime}, \sigma_{f} \cap \mathcal{P}_{d}^{\prime}\right]$, where $\alpha \mid \mathcal{K}_{d}^{\prime} \mapsto \mathbb{R}^{d}$ is some measurable mapping with $\alpha(K+x)=\alpha(K)+x$ for all $x \in \mathbb{R}^{d}$ and $K \in \mathcal{K}_{d}^{\prime}$, for example $\alpha(K)=$ lex $\max (K)$ in what follows. From the theory of stationary marked point process, see Chapt. 3.2 in [19] or [4], we use the factorization of the intensity measure $\mathbf{E} \Psi_{\alpha}(\cdot)$ which implies the existence of a unique probability measure

$$
\widehat{P}_{\mathbf{o}}(\mathcal{A})=\frac{1}{\gamma_{d}} \mathbf{E} \Psi_{\alpha}\left([0,1)^{d} \times \mathcal{A}\right) \quad \text { for } \quad \mathcal{A} \in \sigma_{f} \cap \mathcal{P}_{d, \mathbf{o}}^{\prime}
$$


concentrated on $\mathcal{P}_{d, \mathbf{o}}^{\prime}:=\left\{C \in \mathcal{P}_{d}^{\prime}: \operatorname{lex} \max (C)=\mathbf{o}_{d}\right\}$ with the intensity $\gamma_{d}:=\mathbf{E} \#\{i \geq 1$ : lex $\left.\max \left(Z_{i}\right) \in[0,1)^{d}\right\}$. Now, we are ready to formulate the announced properties of $Z_{\mathbf{o}}$ and $\widehat{Z}_{\mathbf{o}}$ :

Theorem 3.1. Under the assumptions made at the beginning of Sect. 3, it holds:

1. The distribution $P_{\mathbf{o}}$ of the zero cell $Z_{\mathbf{o}}$ does not depend on the distribution of $\Xi_{0}$.

2. For any translation-invariant functional $h: \mathcal{P}_{d}^{\prime} \mapsto[0, \infty)$ the expectation $\mathbf{E} h\left(\widehat{Z}_{\mathbf{o}}\right)=\int_{\mathcal{P}_{d, \mathbf{o}}^{\prime}} h(C) \widehat{P}_{\mathbf{o}}(\mathrm{d} C)$ does not depend on the distribution of $\Xi_{0}$.

Proof. For all $i \geq 1$, the sets $Z_{i}$ and thus the zero cell are regular closed RACS, i.e. $Z_{\mathbf{o}}=$ cl(int $\left.Z_{\mathbf{o}}\right) \mathbf{P}$-a.s. As shown in [18], Chapt. 1.4.2, the distribution $P_{\mathbf{o}}$ is therefore determined if the inclusion functional $I(L):=P_{\mathbf{o}}\left(\left\{F \in \mathcal{F}_{d}: L \subseteq F\right\}\right)$ is known for every finite set $L$. By the definition (3.2) and $P_{\mathbf{o}}^{*}\left(\left\{F \in \mathcal{F}_{d}: \mathbf{o}_{d} \in F\right\}\right)=\mathbf{P}\left(\mathbf{o}_{d} \notin \Xi_{\lambda, Q_{d, d-1}}\right)=1-p(0)=$ $\exp \left\{-\lambda \mathbf{E} \nu_{1}\left(\Xi_{0}\right)\right\}$, it follows that

$$
\begin{aligned}
I(L)=P_{\mathbf{o}}\left(\left\{F \in \mathcal{F}_{d}: L \subseteq F\right\}\right) & =P_{\mathbf{o}}^{*}\left(\left\{F \in \mathcal{F}_{d}: L \subseteq F, \mathbf{o}_{d} \in F\right\}\right) /(1-p(0)) \\
& =\mathbf{P}\left(L \subseteq Z_{\mathbf{o}}^{*}, \mathbf{o}_{d} \notin \Xi_{\lambda, Q_{d, d-1}}\right) /(1-p(0)) .
\end{aligned}
$$

Since $Z_{\mathbf{o}}^{*} \in \mathcal{P}_{d}^{\prime}$ iff $\mathbf{o}_{d} \notin \Xi_{\lambda, Q_{d, d-1}}$, it is obvious that $L \subseteq Z_{\mathbf{o}}^{*}$ for a finite set $L$ implies that $Z_{\mathbf{o}}^{*}$ contains the convex hull $C_{L}:=\operatorname{conv}\left(L \cup\left\{\mathbf{o}_{d}\right\}\right)$ and vice versa. Hence, it suffices to show that $I\left(C_{L}\right)$ does not depend on the distribution of $\Xi_{0}$. It is immediately clear that $C_{L} \subseteq Z_{\mathbf{o}}^{*}$ iff the relative interior relint $\left(C_{L}\right)$ of the polytope $C_{L}$ is contained in the $\left(\mathbf{P}\right.$-a.s.) open set $\Xi_{\lambda, Q_{d, d-1}}^{c}$. Further, due to the stationarity of the P- $(d-1)-\mathrm{CM} \Xi_{\lambda, Q_{d, d-1}}$, the probability that at least one of the at most \#L+1 vertices of $C_{L}$ lies in the boundary $\partial \Xi_{\lambda, Q_{d, d-1}}$ is zero so that the events $\left\{C_{L}=\operatorname{cl}\left(\operatorname{relint}\left(C_{L}\right)\right) \subset \Xi_{\lambda, Q_{d, d-1}}^{c}\right\}$ and $\left\{C_{L} \subseteq Z_{\mathbf{o}}^{*}\right\}$ have the same probability. Therefore, by applying (1.4) and noting that $\pi_{1}\left(-\Theta_{0}^{T} C_{L}\right)$ is an interval, we have

$$
\begin{aligned}
\mathbf{P}\left(C_{L} \subseteq Z_{\mathbf{o}}^{*}, \mathbf{o}_{d} \notin \Xi_{\lambda, Q_{d, d-1}}\right) & =\mathbf{P}\left(C_{L} \cap \Xi_{\lambda, Q_{d, d-1}}=\emptyset\right)=1-T_{\lambda, Q_{d, d-1}}\left(C_{L}\right) \\
& =(1-p(0)) \exp \left\{-\lambda \mathbf{E} \nu_{1}\left(\pi_{1}\left(-\Theta_{0}^{T} C_{L}\right)\right)\right\} .
\end{aligned}
$$

This combined with (3.4) gives $I(L)=I\left(C_{L}\right)=\exp \left\{-\lambda \mathbf{E} \nu_{1}\left(\pi_{1}\left(-\Theta_{0}^{T} C_{L}\right)\right)\right\}$ for any finite set $L \subset \mathbb{R}^{d}$. Thus, the first part of Theorem 3.1 is proved.

To prove the second part, we note that the intensity $\gamma_{d}$ of $\Psi_{\alpha}$ with $\alpha\left(Z_{i}\right)=\operatorname{lex} \max \left(Z_{i}\right)$ can be expressed as product $\gamma_{d}=(1-p(0)) \nu_{d}\left(Z\left(\lambda, Q_{0}^{(d)}\right)\right)$, where

$$
\nu_{d}\left(Z\left(\lambda, Q_{0}^{(d)}\right)\right)=\frac{\lambda^{d}}{d !} \int_{\left(S^{d-1}\right)^{d}}\left|\operatorname{det}\left(u_{1}, \ldots, u_{d}\right)\right| Q_{0}^{(d)}\left(\mathrm{d} u_{1}\right) \cdots Q_{0}^{(d)}\left(\mathrm{d} u_{d}\right)
$$


and $Z\left(\lambda, Q_{0}^{(d)}\right)$ denotes the associated zonoid connected with a stationary Poisson hyperplane process with intensity $\lambda$ and spherical directional distribution $Q_{0}^{(d)}$, see [19]. A detailed proof of the above shape of $\gamma_{d}$ can be found among others in [2], see also [1]. Now, for any translationinvariant functional $g: \mathcal{P}_{d}^{\prime} \mapsto[0, \infty)$ we integrate $g(\cdot)$ w.r.t. the probability measure (3.2). For doing this, we need to apply the Campbell theorem for stationary marked point processes, see Chapt. 3.5 in [19], which implies that

$$
\begin{aligned}
\mathbf{E} g\left(Z_{\mathbf{o}}\right) & =\frac{1}{\mathbf{P}\left(\mathbf{o}_{d} \notin \Xi_{\lambda, Q_{d, d-1}}\right)} \mathbf{E}\left[\sum_{i \geq 1} 1\left(\mathbf{o}_{d} \in \operatorname{int}\left(Z_{i}\right)\right) g\left(Z_{i}\right)\right] \\
& =\frac{\gamma_{d}}{1-p(0)} \int_{\mathcal{P}_{d, \mathbf{o}}^{\prime}} \int_{\mathbb{R}^{d}} g(C) 1\left(\mathbf{o}_{d} \in x+C\right) \mathrm{d} x \widehat{P}_{\mathbf{o}}(\mathrm{d} C)=\gamma_{d} \frac{\mathbf{E}\left[g\left(\widehat{Z}_{\mathbf{o}}\right) \nu_{d}\left(\widehat{Z}_{\mathbf{o}}\right)\right]}{1-p(0)}
\end{aligned}
$$

Finally, replacing $g(\cdot)$ by $h(\cdot) / \nu_{d}(\cdot)$ for an arbitrary translation-invariant functional $h: \mathcal{P}_{d}^{\prime} \mapsto$ $[0, \infty)$ reveals that

$$
\mathbf{E} h\left(\widehat{Z}_{\mathbf{o}}\right)=\frac{1-p(0)}{\gamma_{d}} \mathbf{E}\left[h\left(Z_{\mathbf{o}}\right) / \nu_{d}\left(Z_{\mathbf{o}}\right)\right]=\frac{1}{\nu_{d}\left(Z\left(\lambda, Q_{0}^{(d)}\right)\right)} \mathbf{E}\left[h\left(Z_{\mathbf{o}}\right) / \nu_{d}\left(Z_{\mathbf{o}}\right)\right]
$$

The first part of Theorem 3.1 and (3.5) show that the right-hand side of (3.6), and thus also the expectation on the left-hand side, does not depend on the distribution of $\Xi_{0}$. Hence, the proof of Theorem 3.1 is complete.

\section{References}

[1] C. BräU: Studies on Distributional Properties and Asymptotics of Stationary Poisson Cylinder Processes (in German), Dissertation, University of Augsburg, 2015.

[2] C. BräU and L. Heinrich: Central limit theorems for point processes associated with Poisson $(d-1)$-cylinder models in $\mathbb{R}^{d}$, to be submitted in (2016).

[3] S.N. Chiu, D. Stoyan, W.S. Kendall and J. Mecke: Stochastic Geometry and its Applications, 3rd ed., Wiley, Chichester, 2013.

[4] D.J. Daley And D. Vere-Jones: An Introduction to the Theory of Point Processes: Volume II: General Theory and Structure, 2nd ed., Springer, New York, 2008.

[5] P. Davy: Stereology. A Statistical Viewpoint, Dissertation, Australian National University, Canberra, 1978. 
[6] L. HeinRICH: On existence and mixing properties of germ-grain models, Statistics 23 (1992), pp. 271-286.

[7] L. HeinRICH: Large deviations of the empirircal volume fraction for stationary Poisson grain models, Ann. Appl. Probab. 15, No. 1A (2005), pp. 392-420.

[8] L. Heinrich and M. Spiess: Berry-Esseen bounds and Cramér-type large deviations for the volume distribution of Poisson cylinder processes, Lith. Math. J. 49 (2009), pp. 381-398.

[9] L. Heinrich: Asymptotic Methods in Statistics of Random Point Processes, in Stochastic Geometry, Spatial Statistics and Random Fields, E. Spodarev (ed.), Springer, 2013.

[10] L. Heinrich And M. Spiess: Central limit theorems for volume and surface content of stationary Poisson cylinder processes in expanding domains, Adv. Appl. Probab. 45 (2013), pp. 312-331.

[11] O.E. Lanford and D. Ruelle: Observables at infinity and states with short range correlations in statistical mechanics, Comm. Math. Phys. 13 (1969), pp. 194-215.

[12] S. Martínez And W. Nagel: STIT tessellations have trivial tail- $\sigma$-algebra, Adv. Appl. Probab. 46 (2014), pp. 643-660.

[13] S. Martínez and W. Nagel: The $\beta$-mixing rate of STIT tessellations, Stochastics 88 (2016), pp. 396-414.

[14] G. Matheron: Random Sets and Integral Geometry, Wiley, New York, 1975.

[15] P. McMullen: The volume of certain convex sets, Math. Proc. Cambridge Philos. Soc. 91 (1982), pp. 91-97.

[16] R.E. Miles: The random division of space, Adv. Appl. Probab. 4 (1972), pp. 243-266.

[17] R.E. Miles: Synopsis of Poisson flats in Euclidean spaces, in Stochastic Geometry, E.F. Harding, Wiley, London, 1974.

[18] I. Molchanov: Theory of Random Sets, Springer, London, 2005.

[19] R. Schneider And W. WeIL: Stochastic and Integral Geometry, Springer, Berlin, 2008.

[20] M. Spiess And E. Spodarev: Anisotropic Poisson processes of cylinders, Methodol. Comput. Appl. Probab. 13 (2011), pp. 801-819. 